EPJ Web of Conferences 74, 02001 (2014)

DOI: 10.1051/epjconf/ 20147402001

(C) Owned by the authors, published by EDP Sciences, 2014

\title{
A possible scheme for measuring gravitational waves by using a spinful quantum fluid
}

\author{
Yao Cheng \\ Department of Engineering Physics, Tsinghua University, Beijing, China
}

\begin{abstract}
A method is proposed for measuring gravitational waves (GWs) from the collective electromagnetic (EM) response of a spinful quantum fluid, based on recent studies of the long-lived Mössbauer state ${ }^{93 \mathrm{~m}} \mathrm{Nb}$ in a pure $\mathrm{Nb}$ crystal. A pronounced EM response was found for the geometric phase by rotating the sample in a magnetic field, suggesting that GWs could also be detected. It was recently suggested that the macroscopic wave functions confined in two twisted nonspherical superconductors would give a geometrical phase oscillation induced by GWs. The sensitivity to GWs would be inversely proportional to the square of the bound length, which is the detector size. The proposed sensitivity to GWs would be dramatically enhanced by changing the characteristic size, i.e., using the microscopic size of a non-spherical particle instead of the macroscopic detector size of a scalar quantum fluid. The collective EM response from the quantum fluid would allow the macroscopic geometrical phase to be read from microscopic particles. GWs in the millihertz range, with amplitude of $10^{-22}$, would be detectable.
\end{abstract}

\section{Introduction}

Supposing that two clocks running at the same frequency but which are not synchronized are superimposed at the same position (see Figure 1). A weak passing gravitational wave (GW) [1,2] detunes the clock frequencies by coupling to the GW depending on their orientations. GWs with an amplitude of the order of $10^{-22}$ can be measured if three important transducer conditions are met. First, the transducer must have an excellent quality factor of $\mathrm{Q}>>10^{22}$, so that the almost invisible detuning of the clock frequency can be identified. Second, the detector must be sensitive enough to measure the change. Third, the transducer must respond only to spin-2 (quadrupole) GWs, without any influence of noise from spin-1 (dipole) effects, such as vibration, rotation, or EM fields, or from spin-0 (scalar) effects, such as gravitational potential, in order to give an adequate signal-to-noise ratio.

It has been suggested that GWs could be measured using a quantum fluid [2]. The size and costs involved in building a GW detector can be reduced dramatically by using the collective response of the quantum fluid to GWs. The main GW sensing concepts are based on a cross-coupling term between the electromagnetic (EM) and gravitomagnetic potentials. Sorge [3] recently suggested a very different approach, mimicking quadrupole-quadrupole coupling, which is the Linet-Tourrenc term [4] induced by GWs. Low frequency GWs create virtual quadrupole vibrations to give a differential geometric phase between the macroscopic wavefunctions in two superconductors made of anisotropic double-wells, which are orthogonally twisted with respect to each other. The Josephson junction between the superconductors will then show a response to GWs according to the tunnelling current.

This is an Open Access article distributed under the terms of the Creative Commons Attribution License 4.0, which permits unrestricted use, distribution, and reproduction in any medium, provided the original work is properly cited. 
This detector is not free from dipole noise caused by the magnetic flux penetrating the junction. Moreover, the sensitivity of the detector, as the ratio $\Delta \omega_{k} / \omega_{G W}$, where $\Delta \omega_{k}$ is the detuning from a quadrupole vibration and $\omega_{G W}$ is the GW frequency, is inadequate for detecting GWs in the millihertz range and with amplitudes of the order of $10^{-22}$. The detuning $\Delta \omega_{k} \sim 2 \hbar \pi^{2} / m_{e} L^{2}$ is inversely proportional to the effective mass of electrons $m_{e}$ and inversely proportional to the square of the characteristic size $L$, and $\hbar$ is Planck's constant. A typical micrometre-sized transducer gives $\Delta \omega_{k} \sim 10^{8}$. A ratio of $10^{11}$ is therefore insufficient for measuring the available source GWs in the millihertz range. Decreasing $L$ is more effective than decreasing the particle mass in improving the sensitivity of the transducer.

${ }^{103 \mathrm{~m}} \mathrm{Rh}$ and ${ }^{93 \mathrm{~m}} \mathrm{Nb}$ [5] are long-lived Mössbauer transitions, with half-lives of $1 \mathrm{~h}$ and $16 \mathrm{y}$, respectively. These two $\gamma$ transitions are high-Q clocks that are solely controlled by the multipolar transition energy, which is barely affected by the EM dipole agitations. GWs modulate the transitions not only in terms of their lifetimes (the imaginary part, from the off-diagonal term) [5], but also in terms of the tick-tock frequency (the real part, from the diagonal term). Directional coupling between the multipolar transition and the GW quadrupole gives a detuning that depends on the nuclear orientation. Because of their high frequencies of $\sim 10^{19} \mathrm{~Hz}$, the intrinsic Q-values of ${ }^{103 \mathrm{~m}} \mathrm{Rh}\left(10^{23}\right)$ and ${ }^{93 \mathrm{~m}} \mathrm{Nb}\left(10^{28}\right)$ are higher than the required value $\left(10^{22}\right)$. Furthermore, the amplification factor, $\Delta \omega_{k} / \omega_{G W}$, is increased considerably by using a nuclear particle size.

Although ${ }^{103 \mathrm{~m}} \mathrm{Rh}$ and ${ }^{93 \mathrm{~m}} \mathrm{Nb}$ are excellent candidates, fulfilling the conditions necessary to detect GWs, the problem remains of how to read their responses to GWs. Here, we suggest that a quantum liquid of these long-lived $\gamma$-excitations, involving the superimposition of nonspherical clocks with different orientations, will give a differential reading from the collective EM responses.

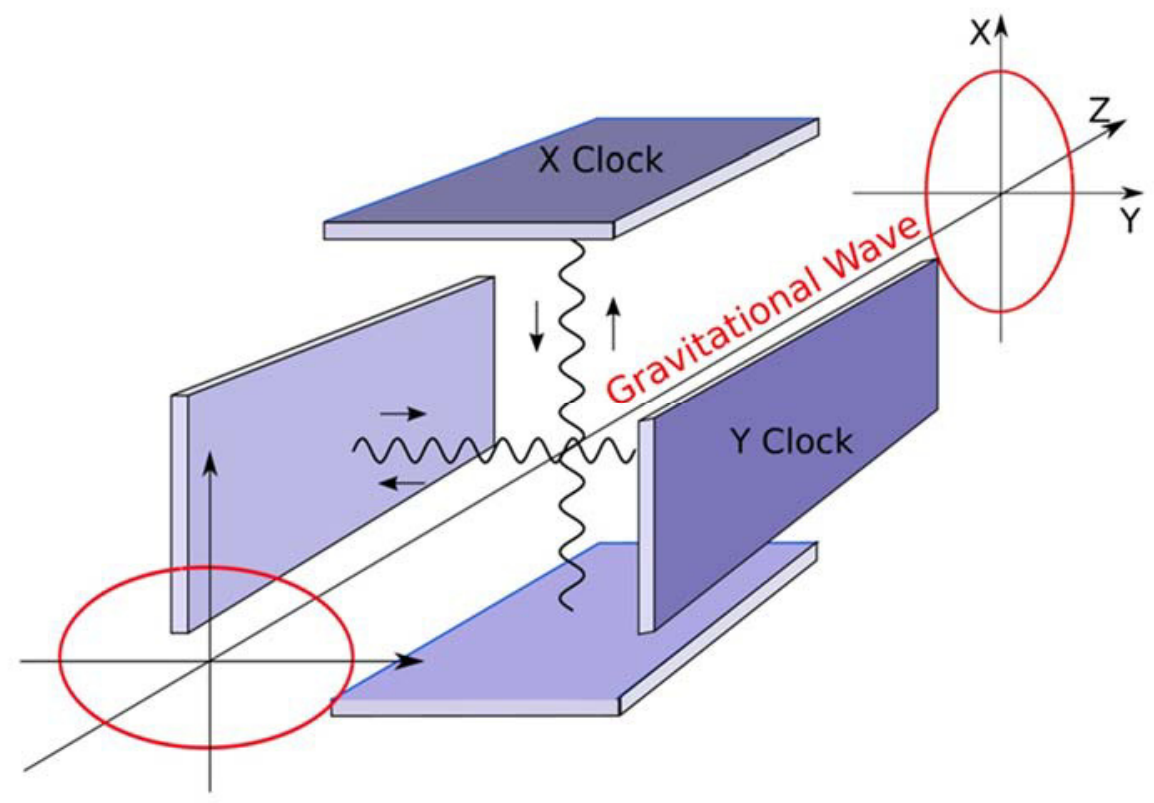

Figure 1. A gravitational wave $(\mathrm{GW})$ detector consisting of two superimposed and identical clocks ticked and tocked by massive standing waves in the $\mathrm{x}$ - and $\mathrm{y}$-directions. A GW, denoted by the red oval, propagates in the $\mathrm{z}-$ direction with tidal actions in the $\mathrm{x}$ - and $\mathrm{y}$ - directions. The $\mathrm{GW}$ will detune the clocks to create a differential phase between the waves confined between walls. 


\section{Nuclear GW detector}

\subsection{Detecting mechanism}

In the classical view, weak GWs propagate spatial curvature ripples [3] that will "deform" a nucleus. The Linet-Tourrenc term for the interaction Hamiltonian is

$$
H_{I}=\frac{1}{2 M} \boldsymbol{P} \stackrel{\leftrightarrow}{h}
$$

where $M$ is the effective mass of the nucleons, $\leftrightarrow$ are the GWs, and $\boldsymbol{P}$ is the momentum operator. The quadrupole wave function deformation is given by the first order perturbation [5]

$$
\delta|n\rangle=h_{ \pm} \sum_{s} \frac{|s\rangle\left\langle s\left|\left(P_{x} \pm i P_{y}\right)^{2}\right| n\right\rangle}{2 M \hbar\left(\omega_{n}-\omega_{s}\right)}+\text { c.c. }
$$

where $h_{ \pm}$are the amplitudes of the two GW polarizations in the transverse traceless (TT) gauge propagating in the $z$-direction. The amplitude of the $\mid \mathrm{s}>$ state, quadruple phonon (roton) excitation [6] contains fractional $x$ - and $y$-contributions in the total transition energy from $\mid \mathrm{n}>$ to $|\mathrm{s}\rangle$. Unlike the dipole deformation caused by an electric field, the quadrupole deformation described by equation 2 is independent of the nuclear size, i.e., the hardness of the nuclear vibration (rotation). The quadrupole phonon (roton) energy is in the mega-electron-Volts range for an atomic mass of around 100 [6].

Assuming the nucleus to be a liquid drop of quantum fluid, Sorge's concept [3] gives rise to a phase shift for a nonspherical nucleus with a characteristic size in the femtometre range. It should be noted that the GW polarization formulations in references [3] and [5] are slightly different. One of the GW polarizations, $h_{\times}=0$, is simply omitted in the present study. The additional phase advance $\Delta \phi$ induced by the GW polarization $h_{+}$takes the form

$$
\frac{\partial \Delta \phi}{\partial t}=\sim h_{+}\left(\omega_{k x}-\omega_{k y}\right)=h_{+} \Delta \omega_{k}(t)
$$

where the detuning $\Delta \omega_{k}(t)$ between the $x$ and $y$ parts of the kinetic frequencies $\omega_{k x}$ and $\omega_{k y}$ is in the mega-electron-Volts range and depends on the nuclear size. Tidal expansion provides the quadrupole phonon (roton) depending on the orientation of a standing nonspherical nucleus. Accumulating the detuning described in equation 3 gives an oscillating geometrical phase shift $\Delta \phi(t)$ driven by GWs.

In reality, heavy nuclei in crystal are very difficult to form a quantum fluid for any pursuant GW effects to be observable. Therefore, we will take the nuclear transition of interest and assume that this forms a quantum fluid. This multipolar case is quite different to the scalar Cooper pairing in a superconductor [3], in which detuning is attributed to the macroscopic wave function confined by the detector shape. Here, the individual wave function of a particle is nonspherical, as discussed in the previous paragraph. The characteristic length $L$ is therefore the particle size rather than the detector size. Taking the GW detuning into account, the clock shift between two transition states can be linearly estimated as

$$
\hbar h_{+} \Delta \omega_{k} \sim\left\langle\Psi_{E x}\left|H_{I}\right| \Psi_{E x}\right\rangle,
$$

where $\left|\Psi_{E x}\right\rangle$ describes a nuclear $\gamma$-excitation delocalized in the crystal of resonant nuclei, i.e., ${ }^{103 \mathrm{~m}} \mathrm{Rh}$ $(40 \mathrm{keV})$ and ${ }^{93 \mathrm{~m}} \mathrm{Nb}(30 \mathrm{keV})$. Two quadrupole energies, for the upper and ground states, almost cancel each other out. The residual quadrupole energy is much less than the mega-electron-Volt range simply because the delocalized size is much greater than the femtometre range. Therefore, we will take another approach, letting the kinetic term equal one half of the exciton energy. $\Delta \omega_{k}$ is of the order of $10^{19} \mathrm{~Hz}$ in this case.

The $\gamma$ quadrupole emission has an angular distribution that depends on the magnetic quanta $\mathrm{m}$ of the nucleus [7], as does the rotation of a nonspherical star emitting GWs. It is natural to take an m-dependent detuned equation 4 from a passing GW plane wave. The amount of detuning depends on the GW 
incident angle, the mechanism of which will be investigated in detail later on, when the spin and the orbital states of the exciton condensate in question become clear. The amplitude of the oscillating phase shift,

$$
\Delta \phi \sim h_{+} \Delta \omega_{k} / \omega_{G W},
$$

for $\mathrm{m}$ quanta approaches unity when estimated for $\Delta \omega_{k} \sim 10^{19} \mathrm{~Hz}$ for GWs in the millihertz range with an amplitude $h_{+} \sim 10^{-22}$. Detailed estimation will be reported by P. Xu in the near future.

\subsection{Geometric phase readout}

A known spin-1 quantum fluid of Cooper pairing ${ }^{3} \mathrm{He}$ provides an example for reading the differential contributions for the m-dependent geometric phases. The macroscopic wave function consists of the superimposition of a spin-up and a spin-down pair,

$$
\Psi=e^{i \phi / 2}|\uparrow \uparrow\rangle+e^{-i \phi / 2}|\downarrow \downarrow\rangle .
$$

The phase $\phi$ can be driven by a time-varying longitudinal magnetic field $B(t)$ [8] and can lead to a longitudinal oscillation with a natural frequency of $\omega_{l}$ oscillating near the balance of $\phi=0$, governed by the pendulum equation

$$
\ddot{\phi}+\omega_{l}^{2} \sin (\phi+\Delta \phi)=\dot{B}(t)
$$

A slow-varying phase shift $\Delta \phi(t)$ induced by GWs modulates the response driven by an alternative magnetic field. It should be noted that the sensitivity of the ${ }^{3} \mathrm{He}$ superfluid to GWs is barely two thousand times higher than the sensitivity of Sorge's superconductive detector to GWs because the mass of ${ }^{3} \mathrm{He}$ is greater than the mass of an electron, although the atomic size is much smaller than the detector size. Therefore, the sensitivity of the ${ }^{3} \mathrm{He}$ superfluid is far from the required level for any notable GW effect.

\subsection{Detector}

The quantum fluids reported here for measuring GWs rely on the outcomes of a long-term research project, begun in 2004, on long-lived Mössbauer isotopes. Even before the physics community is persuaded of the existence of the long-lived Mössbauer effect, it is worth developing the GW detector concept using this possible spinful quantum fluid. The relevant experimental results and their underlying physics are given here, though not in any great detail.

The speculated quantum fluid is a condensate of nuclear $\gamma$-excitation linking two characteristic sizes together to give the possibility of detecting GWs, i.e., with a microscopic nuclear size in the femtometre range for sensing GWs, and a macroscopic wavefunction in the centimetre range for reading the signal.

\section{Long-lived Mössbauer effects}

\subsection{Nuclear $\gamma$ excitation}

The nuclear excitation of ${ }^{45 \mathrm{~m} S c}$ was generated by the bremsstrahlung from a 6-MeV linear accelerator in the Department of Engineering Physics, Tsinghua University, Beijing at the very beginning of this research project. The luminescence of ${ }^{45 \mathrm{~m}} \mathrm{Sc}$ [9] was abnormal, i.e., the time constant of the decay was faster than the published value (half-life $0.3 \mathrm{~s}$ ) [10]. The nuclear luminescence contained a strong 6- 
$\mathrm{keV}$ peak, which was one half of the energy of the 12-keV M2 $\gamma$ transition [10]. The experiment was very difficult to perform because of the short half-life. Our interest then switched to another long-lived Mössbauer nuclide, ${ }^{103 \mathrm{~m}} \mathrm{Rh}$, which has a half-life of $1 \mathrm{~h}$. Two phenomena, luminescence of one half of the $\gamma$ energy and an abnormal decay time at $77 \mathrm{~K}$, were again found.

${ }^{45} \mathrm{Sc}$ and ${ }^{103} \mathrm{Rh}$ are special nuclear species, being the only isotopes of these elements that occur naturally, each having a long-lived low-lying Mössbauer state [10]. Other long-lived Mössbauer nuclides have been studied, and a pure silver sample with the natural abundances of ${ }^{107} \mathrm{Ag}(52 \%)$ and ${ }^{109} \mathrm{Ag}(48 \%)$ did not give the anomalies described above. Therefore, we attributed the anomalies to the fact that ${ }^{45} \mathrm{Sc}$ and ${ }^{103} \mathrm{Rh}$ are present as single isotopes. It was therefore interesting to test another longlived Mössbauer nuclide ${ }^{93} \mathrm{Nb}$, which also has a $100 \%$ natural abundance. The long lifetime of ${ }^{93 \mathrm{~m}} \mathrm{Nb}$ (16 y) allowed us to perform experiments at temperatures below $4 \mathrm{~K}$.

${ }^{93 \mathrm{~m}} \mathrm{Nb}$ has been created in two high-purity $\mathrm{Nb}$ samples using neutron irradiation in the reactor at Tsinghua University, Hsinchu, Taiwan. Some unwanted side effects were found, e.g., the monitoring of $\mathrm{Nb}$ X-rays was affected by interference from radioactive ${ }^{182} \mathrm{Ta}$, caused by Ta impurities activated by neutrons. However, the temperature-dependent decay of ${ }^{93 \mathrm{~m}} \mathrm{Nb}$ was observed, indicating densitydependent decay. Careful studies to verify the abnormal ${ }^{93 \mathrm{~m}} \mathrm{Nb}$ decay time are still ongoing [11], and the results due at the end of 2013, when the interference of ${ }^{182} \mathrm{Ta}$ will be negligible, are expected to be conclusive. So far, the ${ }^{93 \mathrm{~m}} \mathrm{Nb}$ luminescence of one-half of the $\gamma$ energy $(15 \mathrm{keV})$ has been too weak to be observed repeatedly.

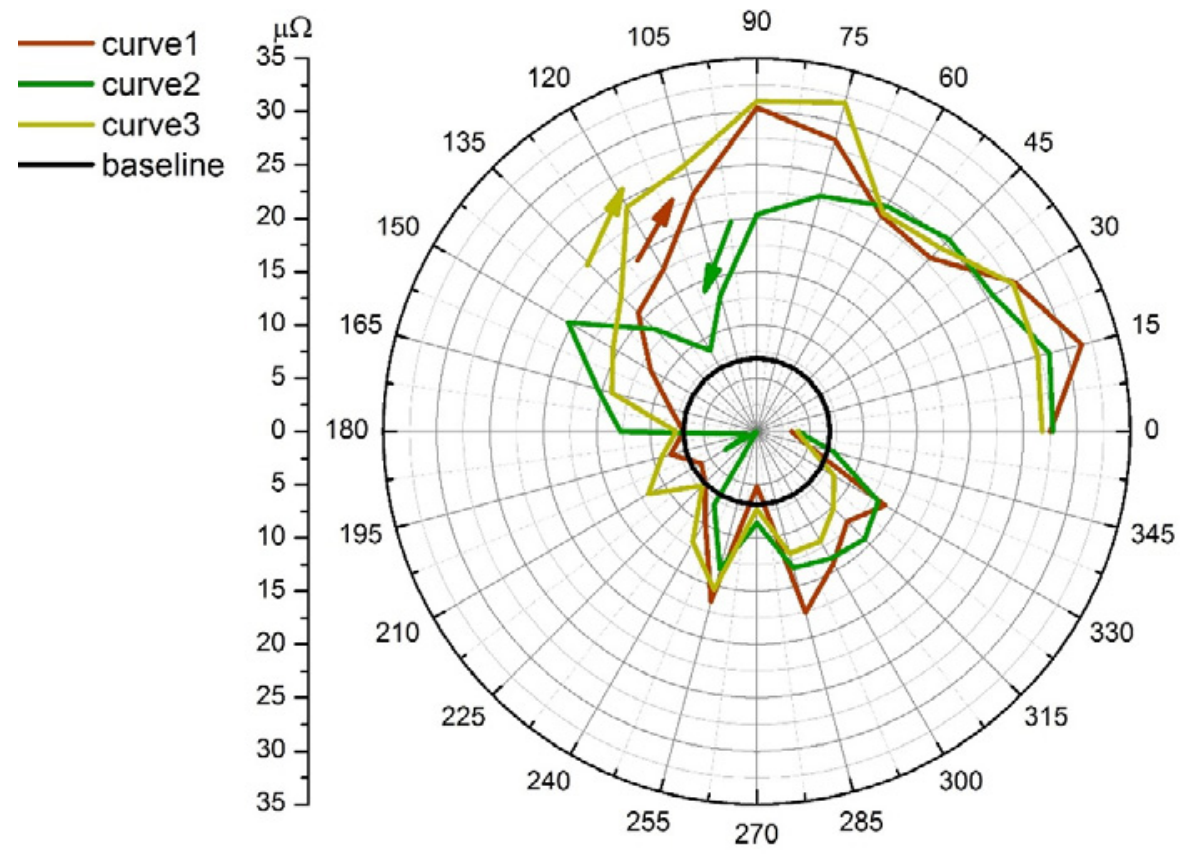

Figure 2. The rotation map of magnetoelectric (ME) response at $4 \mathrm{~K}$ and $9 \mathrm{~T}$. Here, the transverse ME inductance driven by the $200-\mathrm{mA}$ and $20-\mathrm{Hz}$ ac current is presented. The baseline is measured at zero applied field, i.e., the inductive cross coupling between driving and pick-up wires. Curves 1, 2 and 3 show the rotating sequence with clockwise and anticlockwise rotating directions. The ME response starts with a capacitive contribution (below the baseline) at $360^{\circ}$ and arrives an inductive contribution (above the baseline) at $0^{\circ}$. The open response after a single rotation is repeatable.

\subsection{Spinful quantum fluid}

A theory has been suggested to explain the anomalies described above [12]. The multipolar long-lived Mössbauer transition emits two entangled photons (a biphoton) [13], the wavelength of which 
automatically matches the crystal lattice constants to give the collective effect. A crystal consisting of identical nuclei becomes a photonic crystal [13] for the resonant biphoton to stop its propagation. The localized Mössbauer biphoton cannot propagate and obtains a mass. This massive wave carries spin but no charge, and is the so-called nuclear spin-density wave (NSDW).

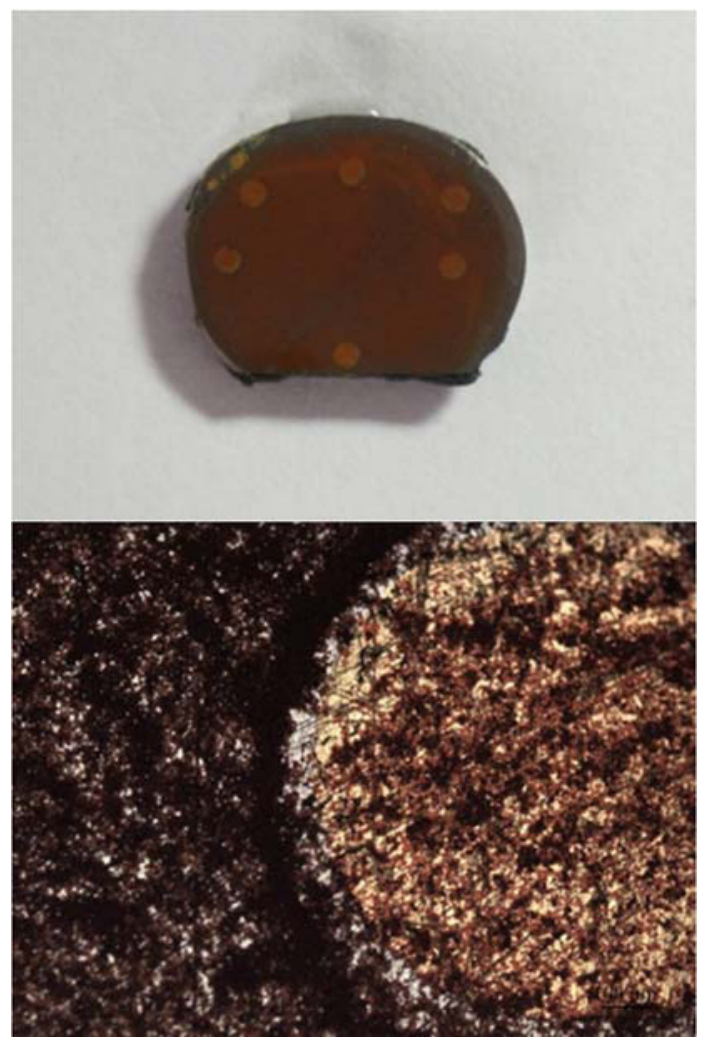

(a)

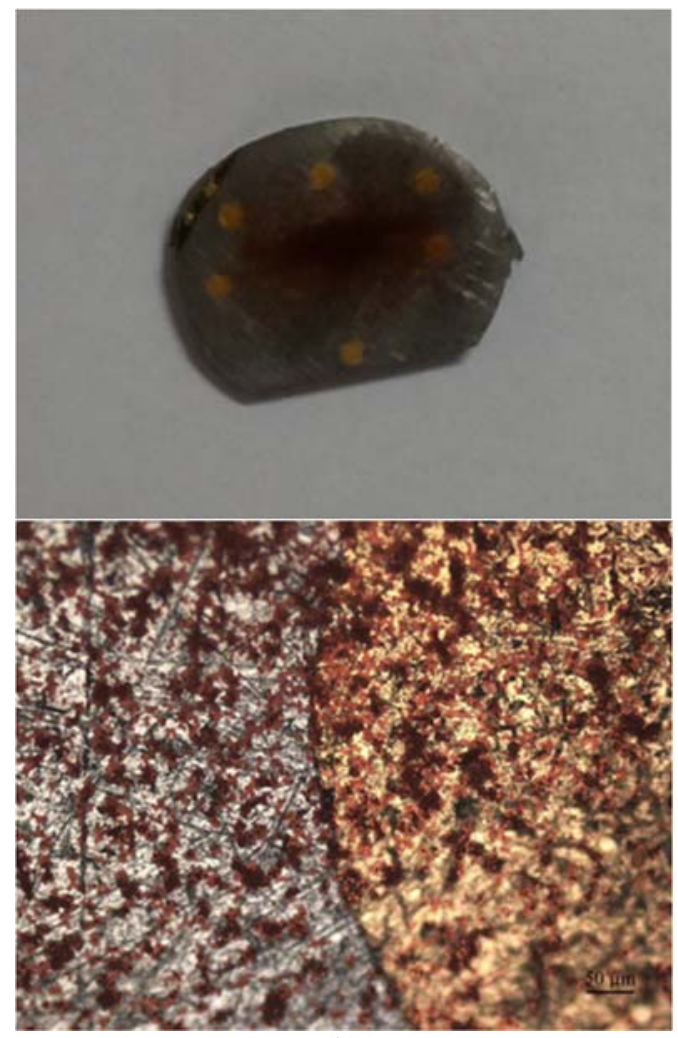

(b)

Figure 3. Photographs of the activated $\mathrm{Nb}$ sample $(1 \mathrm{~cm}$ in diameter $)$ with magnetic beads $(4 \mu \mathrm{m})$ on its surface. The six round yellow spots are gold rods $(\phi 1 \mathrm{~mm})$ that penetrate the $1 \mathrm{~mm}$ thick sample and are used as connectors for measuring the magnetoelectric response. The activated $\mathrm{Nb}$ sample was first heated to $400 \mathrm{~K}$ at $9 \mathrm{~T}$, and then the picture (a) were taken at room temperature $(\sim 300 \mathrm{~K})$, while the pictures (b) were taken three days after. (a) Magnetic beads spread all over the sample, accumulating at the field gradient (i.e., the interface between $\mathrm{Nb}$ and gold), which is revealed by the blow-up figure below. This is evidence that the NSDW is topologically nontrivial, trapping the magnetic fluxes in the sample, especially in the gold rods, which are topological defects. (b) are contrasting pictures to prove that the nonmagnetic $\mathrm{Nb}$ metal loses its capacity to trap magnetic fluxes. The magnetic beads gather together at the centre of the sample surface because of the magnetic attraction between them. The image near the edge of the gold rod is clear (compared to the blow-up picture of (a)), the orange particles being the magnetic beads.

The NSDW undergoes Bose-Einstein condensation (BEC) when the NSDW density exceeds a critical value [14]. The collapse and revival of the ${ }^{103 \mathrm{~m}} \mathrm{Rh}$ spectra occurs when the ${ }^{103 \mathrm{~m}} \mathrm{Rh}$ density becomes greater than a critical value of $10^{12} \mathrm{~cm}^{-3}$. The $\mathrm{Nb}$ sample also shows a pronounced magnetoelectric (ME) effect [15], possibly caused by the collective spin dynamics, when the density of ${ }^{93 \mathrm{~m}} \mathrm{Nb}$ exceeds the similar critical value of $10^{12} \mathrm{~cm}^{-3}$.

The ME response increases with the applied field. When we rotate the sample one full turn of $360^{\circ}$ under an applied field, the transverse $\mathrm{ME}$ response does not return to the initial value but rather gives a pronounced open-loop response to register the winding number, as shown in figure 2 . The value of open-loop response is independent of the advancing angular steps, which demonstrates the well-known characteristic of a geometric phase contribution [16]. 
The activated $\mathrm{Nb}$ sample is a nonmagnetic metal without any significant magnetic contamination, proved by the absence of the Knodo effect [17]. Figures 3 show that the topological nontrivial structure of a NSDW condensate can trap magnetic fluxes.

It is therefore suggested that the ${ }^{103 \mathrm{~m}} \mathrm{Rh}$ and ${ }^{93 \mathrm{~m}} \mathrm{Nb}$ NSDWs become quantum fluids at room temperature with odd parities, with 3 and 4 spins, respectively, when their excitation density is more than the threshold value of $10^{12} \mathrm{~cm}^{-3}$.

\subsection{Texture}

Texture appears in a quantum fluid with an orbital angular momentum $1>1$, as demonstrated in Cooper pairing ${ }^{3} \mathrm{He}$ [8]. The $\gamma$ emission from ${ }^{103 \mathrm{~m}} \mathrm{Rh}$ depends on a weak applied magnetic field in the Gauss range and the sample shape at room temperature [18], revealing several facts. First, the NSDW has a magnetic chain texture. Second, the individual nuclear orientation is dominated by the NSDW. Third, the NSDW magneton is huge, caused by the delocalization. Therefore, the NSDW quantum fluids of the E3 ${ }^{103 \mathrm{~m}} \mathrm{Rh}$ transition are likely to have an orbital angular momentum 1 , and it is likely that $1=1$ when $\mathrm{s}=2$.

Similarly, it is expected that the NSDW quantum fluids of the ${ }^{93} \mathrm{~m}$ Nb M4 transition may contain an orbital angular momentum and therefore a texture. The anisotropic magnetoelectric response of the ${ }^{93 \mathrm{~m}} \mathrm{Nb} \mathrm{NSDW}$ (see Fig. 2) [15], which depends on the sample shape and the direction of the applied field, supports this.

\section{Low-frequency signals}

Strange low-frequency signals containing several clear peaks, e.g., at $\sim 1.3$ and $\sim 6 \mathrm{mHz}$, were observed in the time-varying broadening of the characteristic ${ }^{103 \mathrm{~m}} \mathrm{Rh}$ luminescence when the highly activated sample (at an excitation density $>10^{12} \mathrm{~cm}^{-3}$ ) was cooled to $77 \mathrm{~K}$ using liquid nitrogen. The peak amplitudes were not stable from one measurement to another over a period of one week. However, the $6-\mathrm{mHz}$ peak was observed several times. The signal was not sensitive to ground vibrations or to the 'jittering' of the earth's magnetic field. No characteristic water and air tidal wave peaks appeared in the millihertz range. This observation led to the speculation that the signal is induced by GWs. A simple analysis [5], aimed at describing the interactions between multipolar nuclear transition and GWs, did not provide sufficient sensitivity to allow the possible signal from GWs to be understood.

The strange signal at $\sim 6 \mathrm{mHz}$ appeared again in 2011 when the $\mathrm{Nb}$ sample containing ${ }^{93 \mathrm{~m}} \mathrm{Nb}$ was cooled to $3 \mathrm{~K}$ without applying a field. The superconductor should not give any voltage response to the alternating current drive. However, a pronounced 6-mHz modulation on the 1.1-Hz drive was observed. At the end of 2012 we measured the geometrical phase by rotating the $\mathrm{Nb}$ sample under an applied magnetic field (see Fig. 2) [15]. This geometric phase observation led to the idea that we could detect GWs, as suggested in this paper.

\section{Conclusions}

To gain sufficient sensitivity from a Sorge's superconductive GW detector, the size must be reduced to the subatomic range. Therefore, a nuclear-type GW detector is proposed, where the sensitivity is enhanced by introducing the characteristic nuclear size. The interaction between GW and multipolar transition depends on the GW incident angle, which gives different geometric phase contributions among the macro waves of different magnetic quanta. It is known that the differential phase is then detectable via the internal Josephson effect [8] of a spinful quantum fluid. Our studies of a ${ }^{93 \mathrm{~m}} \mathrm{Nb} \mathrm{NSDW}$ condensate provide a possible solution to detect GWs $\sim \mathrm{mHz}$ with an amplitude of $10^{-22}$.

\section{References}


1. C. W. Misner, K. S. Thorne, and J. A. Wheeler, Gravitation (Freeman, San Francisco, 1973)

2. C. Kiefer and C. Weber, Ann. Phys. 14, 253-278 (2005)

3. F. Sorge, Class. Quantum Grav. 27, 225001-225012 (2010)

4. C. J. Borde, COMPTES RENDUS PHYSIQUE, 2, 509 (2001)

5. Y. Cheng and J. Q. Shen, arXiv:gc-qc/0703066 (2007)

6. K. S. Krane, Introductory Nuclear Physics. (John Wiley \& Sons, Inc., Hoboken, 1988).

7. J. D. Jackson, Classical Electrodynamics. (John Wiley \& Sons, Inc., 1999)

8. A. J. Leggett, Quantum Liquid. (Oxford University Press, New York, 2006)

9. B. Xia, Phd thesis (in Chinese), Department of Engineering Physics, Tsinghua University, Beijing (2007)

10. R. B. Firestone, Ed. Table of Isotopes, 8th Eds. (John Wiley \& Sons, New York, 1999)

11. B. Yang, Student thesis (in Chinese), Department of Engineering Physics, Tsinghua University, Beijing (2013)

12. Y. Cheng, arXiv:0907.1446, (2009)

13. M. Fox, Quantum Optics. (Oxford University Press, New York, 2006)

14. Y. Cheng, arXiv:0906.5417, ICAME, Vienna, Austria (2009)

15. Y.-S. Wang, Student thesis (in Chinese), Department of Engineering Physics, Tsinghua University, Beijing (2013)

16. M. V. Berry, Proc. Roy. Soc. London A 392, 45 (1984)

17. J. Kondo, Prog. Theor. Phys. 32, 37-49 (1964)

18. Y. Cheng \& B. Xia, arXiv:0908.0628, (2009) 